\title{
Postoperative analgesic effectiveness of bilateral erector spinae plane block for adult cardiac surgery: a randomized controlled trial
}

\author{
๑Bülent Barış Güven, ĐTuna Ertürk, @Ayşin Ersoy \\ University of Health Sciences, Sultan 2. Abdulhamid Han Training and Research Hospital, Department of Anesthesia and Reanimation, İstanbul, Turkey
}

Cite this article as: Güven BB, Ertürk T, Ersoy A. Postoperative analgesic effectiveness of bilateral erector spinae plane block for adult cardiac surgery: a randomized controlled trial. J Health Sci Med 2022; 5(1): 150-155.

\begin{abstract}
Introduction: There are few randomized controlled trials examining the effectiveness of bilateral Erector Spinae Plane Block (ESP) with patients undergoing cardiac surgery. The effect of bilateral ESP block on postoperative pain levels and analgesic consumption for patients undergoing open-heart surgery was examined in this single-blind, randomized, controlled trial.

Material and Method: 54 patients who underwent cardiac surgery with open median sternotomy under general anesthesia between May 2020 and June 2021 were included in the study. Patients were randomized into two groups, each consisting of 27 patients, one with $40 \mathrm{ml}$ of $0.25 \%$ bupivacaine and bilateral ESP block, the other with no block implementation (control group). Demographic data, operation type, length of stay in the Intensive Care Unit (ICU), numerical rating scale (NRS) values in the first 24 hours after extubation, and morphine consumption values of the patients were recorded for the study.

Results: In the study, 4 patients were excluded from follow-up due to prolonged intubation in the postoperative period. The data of a total of 50 patients (ESP group $n=25$; Control group $n=25$ ) were analyzed. The duration before first analgesic (mean \pm SD: $459.2 \pm 92.8 \mathrm{~min}$.) of the ESP group was statistically longer than those in the control group (mean \pm SD: $142.0 \pm 56.6$ min.) ( $<<0.001)$. The total length of stay in the ICU and rate of nausea-vomiting were lower for the ESP group than for the control group $(\mathrm{p}<0.05)$. Total morphine consumption (mean \pm SD: $5.1 \pm 3.1 \mathrm{mg}$ ) of the ESP group in the first 24 hours was statistically lower $(\mathrm{p}<0.001)$ compared to the consumption of the control group (mean $\pm \mathrm{SD}: 14.8 \pm 4.2 \mathrm{mg}$ ). NRS scores were significantly lower for the ESP group at postoperative $1^{\text {st }}, 2^{\text {nd }}, 4^{\text {th }}, 6^{\text {th }}$, and $8^{\text {th }}$ hours compared to the control group ( $\mathrm{p}<0.05$ ).

Conclusion: ESP block applied bilaterally in adult cardiac surgeries decreased postoperative pain scores and morphine consumption. At the same time, it was observed that the length of stay of the patients in the ICU was reduced and there were no complications.
\end{abstract}

Keywords: Erector spinae plane block, cardiac surgery, postoperative pain, ultrasound guidance, fascial plane block

\section{INTRODUCTION}

In order to reduce hospital stay after open heart surgeries and improve clinical outcomes, multimodal and multidisciplinary approaches that standardize perioperative care are targeted for development (1). The most important of these approaches developed with enhanced recovery after surgery (ERAS) protocols is to provide adequate pain control with a multimodal approach in order to reduce cardiac-pulmonary complications and postoperative opioid consumption. A multimodal pain management plan should include nonopioid systemic analgesic agents, regional anesthesia techniques, and minimal use of opioids (2).

Thoracic epidural analgesia (TEA), one of the regional anesthesia techniques, has been widely used for cardiac surgeries and has been shown to be effective to relieve postoperative pain (3). However, the risk of possible development of neuraxial hematoma due to perioperative heparinization and the high incidence of hypotension limits the use of TEA. Paravertebral block (PVB) has been applied bilaterally for sternotomy pain for cardiac surgeries, and it has been reported that it reduces the need for opioids in the postoperative period by reducing pain scores and is safer than TEA due to heparinization $(4,5)$. However, there are risks associated with PVB such as pneumothorax, vascular 
injection, and intrathecal injection. The erector spinae plane (ESP) block, which has less such risks and is more easily applicable, is a new interfacial plane block that has been studied a lot recently and is promising in this regard.

It has been shown in many studies that ultrasoundguided ESP block is effective and can be applied safely in chest, abdominal, vertebral, and lower extremity surgeries (6). There are very few randomized controlled trials in the literature examining the effectiveness of ESP block for patients undergoing cardiac surgery. For this single-blind, randomized, controlled trial, it was aimed to examine the effect of bilateral ESP block on postoperative analgesic consumption and pain levels of patients undergoing open heart surgery.

\section{MATERIAL AND METHOD}

This study was designed as a prospective, randomized, single-blind, controlled trial and the study was approved by University of Hamidiye Clinical Research Ethics Committee (Date: 27.02.2020, Decision No: 2020.02.27-17). All procedures were carried out in accordance with the ethical rules and the principles of the Declaration of Helsinki.

The inclusion criteria for 66 patients were: being 18 65 years old, being identified as American Society of Anesthesiologists (ASA) physical status classes I or II, having body mass index (BMI) 20 to $30 \mathrm{~kg} / \mathrm{m}^{2}$, undergoing any of the coronary artery bypass graft (CABG), atrial septal defect (ASD) repair and heart valve replacement operations by opening a median sternotomy under general anesthesia (Figure 1). Patients who underwent two or more surgical procedures in the same operation were not included in the study due to the concern that such combined procedures may increase the duration of the operation and cause differences. Patients with emergency surgery, ASA-3 and above, spinal deformities, failed blocks, bleeding diathesis, local anesthesia allergy, neurological deficit, chronic painkillers, or narcotic drug use as well as patients taking antithrombotic drugs and patients with abnormal coagulation parameters were excluded from the study.

One day before the operation, the patients were informed about the use of the ESP block, the 11-point numerical pain rating scale (NRS), and patient-controlled analgesia (PCA) device, and their written informed consent was obtained in this regard. The patients were randomized using the closed-envelope method and divided into two groups (1:1 allocation ratio). All patients were premedicated with 0.03 mg.kg-1 midazolam on the day of surgery; and then, the patients were taken to the operating room and standard monitoring including $\mathrm{SpO}_{2}$, ECG, non-invasive blood pressure monitoring was applied to all patients. Before general anesthesia, patients in the ESP group were placed in a sitting position for the ESP block and the procedure area was sterilized. A $10-5 \mathrm{MHz}$ Linear probe and an 80 mm B.Braun Stimuplex peripheral block needle were used under the guidance of real-time USG (SonoSite M-Turbo). The USG probe was placed approximately $3 \mathrm{~cm}$ lateral to the T5 spinous process, in the parasagittal plane. After local infiltration with $2 \%$ lidocaine, the block needle was advanced in the craniocaudal direction with an in-plane approach at an angle of 30 degrees from the skin. When the needle reached the $\mathrm{T} 5$ transverse process by passing the trapezius, rhomboid major and erector spinae muscles, $1 \mathrm{ml}$ of normal saline was injected (hydrodissection) between the erector spinae muscle fascia and the transverse process. After confirming the location of the needle in this way, ESP block was applied by administering $20 \mathrm{ml}$ of $0.25 \%$ bupivacaine to the same plane after negative blood aspiration (Figure 2). This process was applied to the other side in the same way. All of the ESP blocks in the study were performed by the same anesthesiologist (B. B. G.). ESP block was not applied to the patients in the control group. General anesthesia was induced by intravenous (IV) administration of $1 \mu \mathrm{g} . \mathrm{kg}^{-1}$ fentanyl, 0.15 mg. $\mathrm{kg}^{-1}$ midazolam, $1-2 \mathrm{mg} \cdot \mathrm{kg}^{-1}$ propofol and $1 \mathrm{mg} \cdot \mathrm{kg}^{-1}$ rocuronium to the patients of both groups. Maintenance of anesthesia was achieved with inhaled sevoflurane in an air-oxygen mixture with $50 \%$ inspired oxygen concentration, rocuronium infusion (0.01 mg.kg. $\left.\mathrm{min}^{-1}\right)$ and fentanyl infusion (2-3 $\left.\mu \mathrm{g} \cdot \mathrm{kg} \cdot \mathrm{h}^{-1}\right)$. At the end of the operation, $1 \mathrm{~g}$ IV paracetamol, $100 \mathrm{mg}$ IV tramadol and 8 $\mathrm{mg}$ IV ondansetron were administered to all patients. After the operation, IV PCA device prepared with morphine $(0.5 \mathrm{mg} / \mathrm{ml}$ concentration, $1 \mathrm{mg}$ bolus dose, 10 min lockin time) was inserted for patients of both groups after the operation. The nurses who filled out the patient follow-up form in the postoperative period were blind to the study and routinely administered $1 \mathrm{~g}$ IV paracetamol every 8 hours in the postoperative period to all of the patients. For patients with NRS $>4,1 \mathrm{mg}$ morphine IV bolus was administered via PCA as a rescue analgesic dose.

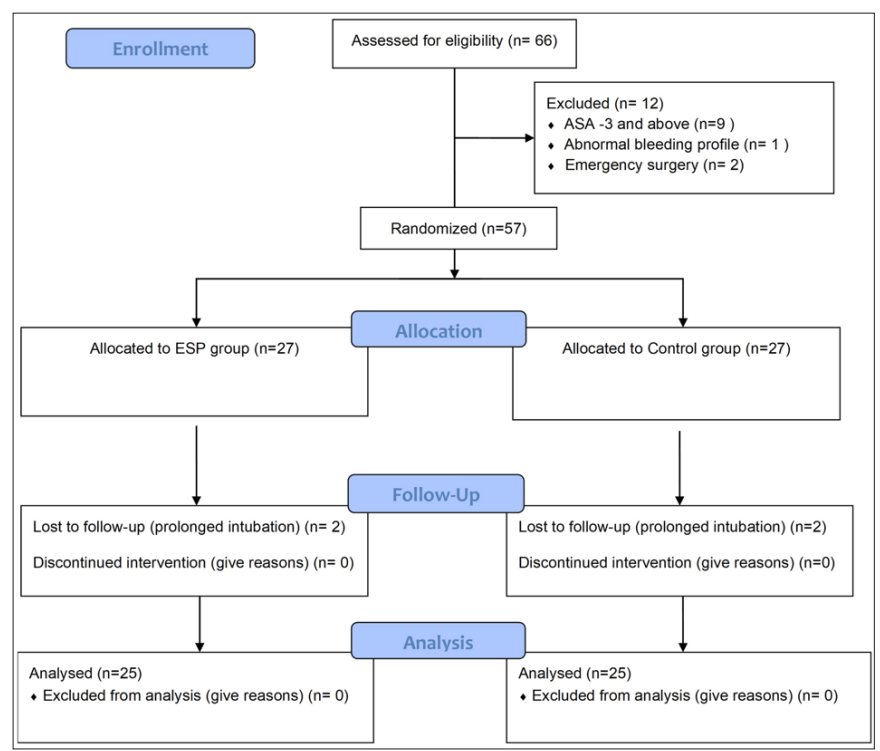

Figure 1. CONSORT Flow Diagram 


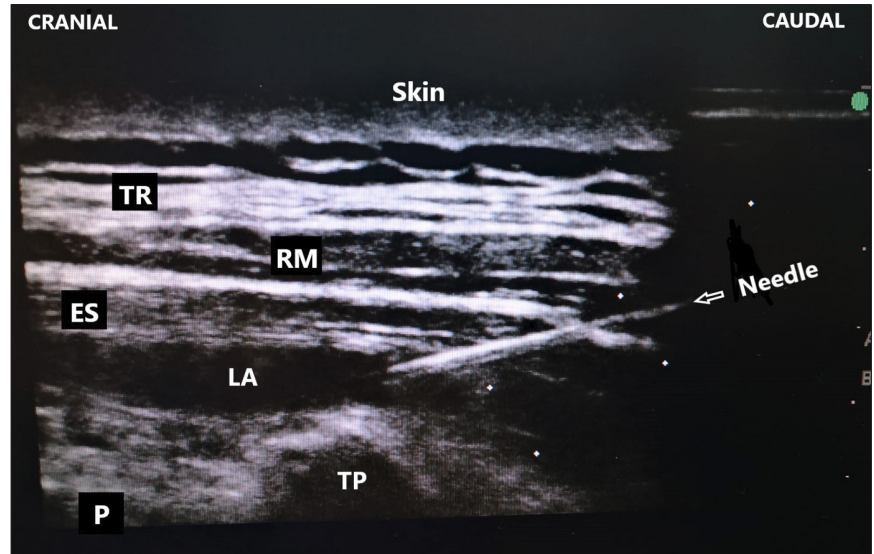

Figure 2. Ultrasound-guided erector spinae plane (ESP) block. TR: Trapezius muscle; RM: Rhomboid major muscle; ES: Erector spinae muscle; LA: Local anesthetic drug; TP: Transverse process; P: Pleura

Hourly and total analgesic consumption during the postoperative 24 hours was the primary target in the study. The duration before the first rescue analgesic, the 11-point NRS (0-10, 0 for no pain and 10 for worst pain), mechanical ventilation duration, ICU length of stay, the incidence of postoperative nausea or vomiting (PONV) were considered as secondary targets. NRS scores were recorded at hourly periods of $1,2,4,6,8,10,12,16,20$ and 24 after extubation. In the meantime, local anesthesia toxicity and complications related to ESPB (hematoma, infection and pneumothorax) were recorded.

\section{Statistical Method}

The power analysis of the study was based on the study of Nagaraja et al. (7) on ESP block. The standard effect size was determined as 0.8 with $5 \%$ margin of error and $80 \%$ power, and a total of 66 patients were included in the study. The mean, standard deviation, median, minimum, maximum, frequency, and ratio values were used in the descriptive statistics of the data. The distribution of the variables was measured with the Kolmogorov Smirnov test, and the independent samples $t$ test and the MannWhitney $\mathrm{u}$ test were used in the analysis of quantitative independent data. The chi-square test was used for the analysis of qualitative independent data, and the fischer test was used when the chi-square test conditions were not met. The analysis was carried out using the SPSS 27.0 program.

\section{RESULTS}

66 patients who underwent CABG, ASD repair, aortic valve replacement (AVR), and mitral valve replacement (MVR) operations by opening median sternotomy under general anesthesia in the Cardiovascular Surgery OT of Sultan 2. Abdülhamit Han Training and Research Hospital between May 2020 and June 2021 were included in the study. Of these patients, 9 patients with ASA3 and above, 1 patient who underwent emergency surgery, and
2 patients with abnormal bleeding profile were excluded from the study. 54 patients were randomized and divided into two groups as the ESP applied group $(n=27)$ and the control group $(n=27)$. The data of 4 patients whose postoperative intubation duration was prolonged $(>6$ hours) were not used for the analysis (Figure 1). Thus, the data of a total of 50 patients from 25 patients in both groups were statistically analyzed.

Age, sex distribution, height, weight, BMI, and European System for Cardiac Operative Risk Evaluation-2 (Euro Score-2) values of the patients did not differ significantly ( $p>0.05)$ between the ESP block group and the control group. There was no significant difference $(p>0.05)$ between the two groups in terms of duration of surgery, type of surgical operation, duration of cardiopulmonary bypass (CPB) and intraoperative total fentanyl consumption (Table 1 and Table 2).

\begin{tabular}{|lccc|}
\hline Table 1. Demographic Data & $\begin{array}{c}\text { ESP group } \\
(\mathbf{n}=\mathbf{2 5})\end{array}$ & $\begin{array}{c}\text { Control group } \\
(\mathbf{n}=\mathbf{2 5})\end{array}$ & $\begin{array}{c}\mathbf{p} \\
\text { value }\end{array}$ \\
\hline Characteristic & $50.6 \pm 16.8$ & $58.7 \pm 12.9$ & 0.063 \\
\hline Age (year) & $14 / 11$ & $12 / 13$ & 0.560 \\
Gender (male /female) & $77.2 \pm 16.2$ & $80.8 \pm 14.4$ & 0.415 \\
Weight $(\mathrm{kg})$ & $169.5 \pm 7.7$ & $167.8 \pm 7.0$ & 0.410 \\
Height $(\mathrm{cm})$ & $26.7 \pm 4.3$ & $28.6 \pm 4.1$ & 0.118 \\
BMI $\left(\mathrm{kg} / \mathrm{m}^{2}\right)$ & $157.2 \pm 21.6$ & $160.4 \pm 21.4$ & 0.606 \\
Duration of surgery (min) & $3.1 \pm 3.9$ & $3.0 \pm 4.0$ & 0.648 \\
Euro Score-2 & & & \\
Type of surgery & $16(64 \%)$ & $17(68 \%)$ & 0.765 \\
CABG & $6(24 \%)$ & $5(20 \%)$ & 0.733 \\
MVR & $2(8 \%)$ & $1(4 \%)$ & 1.000 \\
ASD & $1(4 \%)$ & $2(8 \%)$ & 1.000 \\
AVR &
\end{tabular}

Data are expressed as mean \pm standard deviation or number (proportion), Euro Score: European System for Cardiac Operative Risk Evaluation, CABG:Coronary artery bypass graft, MVR: Mitral valve replacement, ASD: Atrial septal defect, AVR: Aortic valve replacement, Min: Minute.

\begin{tabular}{|c|c|c|c|}
\hline Variable & $\underset{(n=25)}{\text { ESP group }}$ & $\begin{array}{c}\text { Control } \\
\text { group } \\
(\mathbf{n}=25)\end{array}$ & $\underset{\text { value }}{p}$ \\
\hline CPB time (min) & $74.1 \pm 17.7$ & $75.6 \pm 18.3$ & 0.760 \\
\hline $\begin{array}{l}\text { IO total fentanyl } \\
\text { consumption (mcg) }\end{array}$ & $548 \pm 89.5$ & $560 \pm 99$ & 0.588 \\
\hline $\begin{array}{l}\text { PO total morphine } \\
\text { consumption in } 24 \text { hour }(\mathrm{mg})\end{array}$ & $5.1 \pm 3.1$ & $14.8 \pm 4.2$ & $0.000^{*}$ \\
\hline $\begin{array}{l}\text { Total duration of MV in the } \\
\text { ICU (min) }\end{array}$ & $254.0 \pm 36.5$ & $326.4 \pm 29.1$ & $0.001^{\star}$ \\
\hline Time to ambulation (hour) & $28.8 \pm 6.7$ & $37.4 \pm 9.6$ & $0.001^{*}$ \\
\hline $\begin{array}{l}\text { Total length of stay in the } \\
\text { ICU (hour) }\end{array}$ & $46.7 \pm 9.0$ & $55.3 \pm 11.8$ & $0.005^{\star}$ \\
\hline $\begin{array}{l}\text { Time to first use of PCA } \\
(\mathrm{min})\end{array}$ & $459.2 \pm 92.8$ & $142.0 \pm 56.6$ & $0.000^{*}$ \\
\hline $\begin{array}{l}\text { Incidence of PONV in } 24 \\
\text { hour }\end{array}$ & $6 / 25$ & $1 / 25$ & $0.042^{*}$ \\
\hline
\end{tabular}


Mechanical ventilation (MV) duration and duration before mobilization in the ICU were found to be significantly shorter for the ESP group compared to the control group $(\mathrm{p}<0.05)$. The mean duration before first analgesic was $459.2 \pm 92.8$ minutes for the ESP group, and 142.0 \pm 56.6 minutes for the control group. This difference was statistically significant $(\mathrm{p}<0.001)$. The total length of stay in the ICU and rate of nausea-vomiting were significantly lower $(\mathrm{p}<0.05)$ for the ESP group compared to the control group (Table 2).

While total morphine consumption in the first 24 hours was $5.1 \pm 3.1 \mathrm{mg}$ for the patients with ESP block, this consumption was found to be $14.8 \pm 4.2 \mathrm{mg}$ for the control group, and this difference was statistically significant $(\mathrm{p}<0.001)$. Morphine consumption amounts at the postoperative $1^{\text {st }}$ hour, $2^{\text {nd }}$ hour, $4^{\text {th }}$ hour, $6^{\text {th }}$ hour, $8^{\text {th }}$ hour, $12^{\text {th }}$ hour, $16^{\text {th }}$ hour and $20^{\text {th }}$ hour were significantly lower $(\mathrm{p}<0.05)$ for the ESP group compared to the control group. The amount of morphine consumption at the $10^{\text {th }}$ and $24^{\text {th }}$ hours did not differ significantly ( $\mathrm{p}>0.05)$ between the two groups (Table 2 and Table 3 ).

Postoperative $1^{\text {st }}$ hour, $2^{\text {nd }}$ hour, $4^{\text {th }}$ hour, $6^{\text {th }}$ hour, and $8^{\text {th }}$ hour NRS scores of the ESP group were significantly $(p<0.05)$ lower than the scores of the control group (Figure 3). The $10^{\text {th }}$ hour, $12^{\text {th }}$ hour, $16^{\text {th }}$ hour, $20^{\text {th }}$ hour and $24^{\text {th }}$ hour NRS scores did not differ significantly ( $\mathrm{p}>$ 0.05 ) between the two groups (Table 3 ).

\begin{tabular}{|c|c|c|c|}
\hline & ESP group & Control group & p Value \\
\hline \multicolumn{4}{|l|}{ NRS scores } \\
\hline $1^{\text {st }}$ hour & $1.2 \pm 1.3$ & $5.0 \pm 1.7$ & $0.000^{*}$ \\
\hline $2^{\text {nd }}$ hour & $1.2 \pm 1.0$ & $3.5 \pm 1.3$ & $0.000^{*}$ \\
\hline $4^{\text {th }}$ hour & $2.1 \pm 1.4$ & $4.2 \pm 1.8$ & $0.000^{*}$ \\
\hline $6^{\text {th }}$ hour & $2.4 \pm 1.8$ & $4.2 \pm 1.6$ & $0.000^{*}$ \\
\hline $8^{\text {th }}$ hour & $2.2 \pm 1.7$ & $4.1 \pm 1.7$ & $0.001^{*}$ \\
\hline $10^{\text {th }}$ hour & $4.2 \pm 2.2$ & $4.4 \pm 1.3$ & 0.751 \\
\hline $12^{\text {th }}$ hour & $4.5 \pm 1.6$ & $4.6 \pm 1.5$ & 0.842 \\
\hline $16^{\text {th }}$ hour & $4.2 \pm 1.6$ & $4.5 \pm 1.4$ & 0.679 \\
\hline $20^{\text {th }}$ hour & $4.1 \pm 1.2$ & $4.2 \pm 1.3$ & 0.976 \\
\hline $24^{\text {th }}$ hour & $4.4 \pm 1.2$ & $4.4 \pm 1.1$ & 0.832 \\
\hline \multicolumn{4}{|c|}{ Morphine consumption (mg) } \\
\hline $1^{\text {st }}$ hour & $0.2 \pm 0.5$ & $2.8 \pm 1.2$ & $0.000^{*}$ \\
\hline $2^{\text {nd }}$ hour & $0.2 \pm 0.5$ & $2.0 \pm 0.7$ & $0.000^{*}$ \\
\hline $4^{\text {th }}$ hour & $0.1 \pm 0.3$ & $1.8 \pm 1.1$ & $0.000^{*}$ \\
\hline $6^{\text {th }}$ hour & $0.3 \pm 0.5$ & $1.4 \pm 0.8$ & $0.000^{*}$ \\
\hline $8^{\text {th }}$ hour & $0.2 \pm 0.5$ & $1.2 \pm 0.8$ & $0.000^{*}$ \\
\hline $10^{\text {th }}$ hour & $1.2 \pm 0.9$ & $1.3 \pm 0.6$ & 0.722 \\
\hline $12^{\text {th }}$ hour & $0.6 \pm 0.6$ & $1.1 \pm 0.7$ & $0.008^{\star}$ \\
\hline $16^{\text {th }}$ hour & $0.9 \pm 0.6$ & $1.3 \pm 0.6$ & $0.050^{*}$ \\
\hline $20^{\text {th }}$ hour & $0.9 \pm 0.6$ & $1.3 \pm 0.6$ & $0.018^{\star}$ \\
\hline $24^{\text {th }}$ hour & $0.6 \pm 0.6$ & $0.5 \pm 0.6$ & 0.598 \\
\hline
\end{tabular}

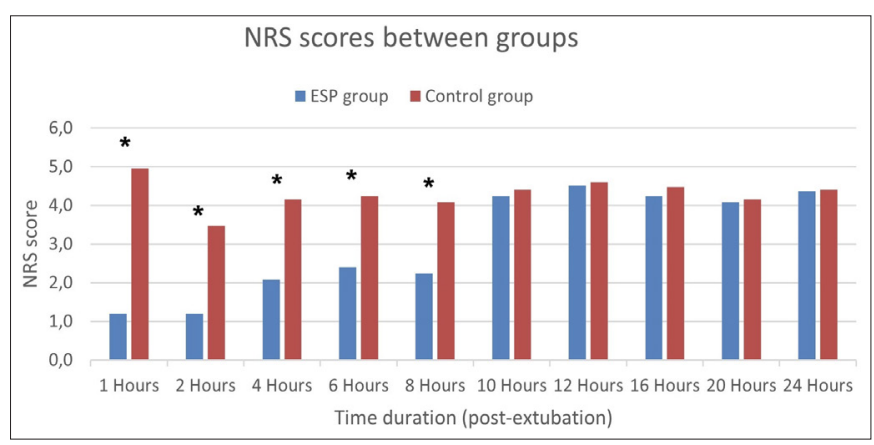

Figure 3. The mean values of the numerical rating scale (NRS) scores between the ESP group and the control group for postoperative pain at different time intervals. $\left({ }^{*} \mathrm{p}<0.05=\right.$ statistical significance)

During the study, no complications such as local anesthesia toxicity or nerve damage, hematoma, pneumothorax, and infection that can be associated with ESP block were observed among patients who underwent ESP block operation.

\section{DISCUSSION}

For this prospective, randomized controlled trial, it was aimed to evaluate the effects of USG-guided bilateral ESP block in terms of postoperative pain level, morphine consumption, ICU length of stay, and complications in CABG, ASD repair, AVR and MVR operations. As a result of this study, a significant decrease was determined for the morphine consumption in the first 24 hours and for the NRS scores in the first 8 hours postoperatively for patients who underwent ESP block operation. Along with this decrease in opioid consumption, a significant decrease was found in ICU length of stay, incidence of nausea-vomiting, and MV durations.

Median sternotomy is the most commonly used incision in open heart surgeries and provides the clearest view for the surgeons. It has been reported that $30-75 \%$ of patients undergoing median sternotomy experience moderate level pain in the postoperative period, and chronic pain syndrome develops for more than $4 \%$ of such patients (8). In cardiac surgery, especially for patients with limited cardiac reserve, postoperative pain may cause poor clinical outcomes by stimulating the sympathetic nervous system. At the same time, opioid drugs used systemically to relieve pain may contribute to this poor outcome with their side effects such as prolonged sedation, respiratory depression, ileus, nausea, and vomiting (9). Although minithoracotomy techniques have been developed recently against such concerns, median sternotomy is still the gold standard incision in cardiovascular surgeries. In order to reduce the postoperative pain of this type of incision, new pain management techniques including TEA, PVB, Parasternal block and pecto-intercostal fascial block have been developed in recent years $(3,4,10,11)$. Since 
the sternum is innervated by T2-T6 intercostal nerves, none of these techniques alone can be expected to produce adequate analgesia for median sternotomy. The application difficulties and complication risks of such methods limit their preference for postoperative analgesia as well. In addition, considering the pain caused by the drains in the thoracic wall after cardiac surgery, a method that can provide analgesia not only in the sternum area but also in the thoracic wall would be more fitting. In this respect, the ESP block is more advantageous due to its multidermatomal analgesia in the anterior and posterior areas of the thoracic wall, its easy application, and its low complication risk; also, the ESP block has been the fascial plane block that has been most studied in the last 3-4 years $(6,7)$.

ESP block has been used safely by many authors for postoperative pain in thorax, abdomen, extremity, and spinal surgeries (6). However, there are a limited number of prospective randomized controlled trials for cardiac surgeries (7,12-14). Krishna et al. (13) applied bilateral ESP block for patients undergoing open heart surgery by administering $20 \mathrm{ml}$ of $0.375 \%$ ropivacaine to each side under USG guidance, and similar to our study, they found a significant decrease in postoperative pain levels, opioid analgesic consumption, extubation duration, and ICU length of stay. Macaire et al. (12) also compared the patient group that did not undergo block operation in cardiac surgery with the patient group that underwent continuous ESP using $0.2 \%$ ropivacaine, and they reported that there was a significant reduction in postoperative morphine consumption and mobilization durations. In our study, we also found that the time from the operation to the first mobilization was statistically significantly shorter for the ESP group in the postoperative period. The difference between the two groups was almost 9 hours.

It has been known for a long time that the use of TEA for patients undergoing cardiac surgery reduces the risk of postoperative supraventricular arrhythmia and respiratory complications (15). However, the most important concern for the use of TEA is that anticoagulant treatments used perioperatively in cardiac surgery facilitate epidural hematoma formation and cause the risk of neurological damage. Nagaraja et al. (7) applied continuous bilateral ESP block with $0.125 \%$ bupivacaine to one group and continuous TEA to another group for postoperative analgesia; they reported that ESP block had similar analgesic effectiveness with TEA and no complications were observed. Some authors have reported that ESP block can be safely applied even in cases where TEA cannot be performed due to thrombocytopenia, anticoagulant therapy, or coagulopathy (16-19). However, the data presented in these reports are quite limited and lack sufficient level of evidence. Therefore, more comprehensive randomized controlled trials are needed to determine the safety and effectiveness of this operation for such patient populations. No significant complications such as hematoma, neurological damage, infection, arrhythmia caused by local anesthesia, or pneumothorax were recorded in our study. In the literature, there are only 2 cases that are reported to have development of pneumothorax associated with ESP block $(20,21)$.

Another method that is effective in postoperative pain control is the IV administration of narcotics with patientcontrolled analgesia. However, it is recommended to reduce the use of narcotics within the scope of ERAS protocols due to the adverse effects of narcotic agents such as excessive sedation, ileus, constipation, severe nausea-vomiting, respiratory depression. In our study, IV PCA and morphine were used as rescue analgesia. In the ESP block group, 24-hour cumulative morphine consumption was observed to be reduced by at least $65 \%$, similar to the study of Athar et al. (14). Along with the decrease in morphine consumption, the incidence of nausea and vomiting was also found to be significantly lower for the ESP group. In addition, it was determined that the duration before the first rescue analgesia use was significantly longer for the ESP group compared to the control group. These results are also consistent with the meta-analysis study of Huang et al. (22) with 590 patients about ESP block.

Few authors reported that sufficient parasternal analgesia did not occur during ESP block operation (23). Since the sensory prick test could not be performed in our study, we could not obtain information about the dermatomal analgesia level of our cases. On the other hand, some studies involving 3D imaging suggested that the local anesthetic solution injected in the ESP block spreads towards the epidural and neural foraminal spaces (24). Anatomically, this indicates that the ESP block should provide sternal analgesia. Further studies are required to clarify this paradoxical result.

The results of this study can be evaluated within some limitations. First, no evaluation for a period longer than postoperative 24 hours could be made since the ESP block was performed as a single shot. The second limitation is that the sensory prick test could not be performed, and dermatomal spread could not be evaluated because the patients received general anesthesia immediately after the ESP block was applied, and some patients were intubated and followed under sedation for about 6 hours in the ICU. 


\section{CONCLUSION}

In our study, it was determined that ESP block applied bilaterally in adult cardiac surgeries decreased postoperative pain scores and morphine consumption. In addition, it was observed that the follow-up times of the patients in MV, their length of stay in the ICU and the duration before first mobilization were shortened. USG-guided ESP block is a safe application because its sonoanatomy is easily recognizable and it is far from risky anatomical structures, and also, its chance of failure is low. For these reasons, we think that ESP block should be included in the perioperative analgesic plan for cardiac surgery patients.

\section{ETHICAL DECLARATIONS}

Ethics Committee Approval: The study was approved by University of Hamidiye Clinical Research Ethics Committee (Date: 27.02.2020, Decision No: 2020.02.2717).

Informed Consent: All patients signed the free and informed consent form.

Referee Evaluation Process: Externally peer-reviewed.

Conflict of Interest Statement: The authors have no conflicts of interest to declare.

Financial Disclosure: The authors declared that this study has received no financial support.

Author Contributions: All of the authors declare that they have all participated in the design, execution, and analysis of the paper and that they have approved the final version.

\section{REFERENCES}

1. Ljungqvist O, Scott M, Fearon KC. Enhanced recovery after surgery: a review. JAMA Surg 2017; 152: 292-8.

2. Markham T, Wegner R, Hernandez N, et al. Assessment of a multimodal analgesia protocol to allow the implementation of enhanced recovery after cardiac surgery: retrospective analysis of patient outcomes. J Clin Anesth 2019; 54: 76-80.

3. Guay J, Kopp S. Epidural analgesia for adults undergoing cardiac surgery with or without cardiopulmonary bypass. Cochrane Database Syst Rev 2019; 3: CD006715.

4. Sun L, Li Q, Wang Q, Ma F, Han W, Wang M. Bilateral thoracic paravertebral block combined with general anesthesia vs. general anesthesia for patients undergoing off-pump coronary artery bypass grafting: a feasibility study. BMC Anesthesiol 2019; 19: 101.

5. Mehta Y, Arora D, Sharma KK, Mishra Y, Wasir H, Trehan N. Comparison of continuous thoracic epidural and paravertebral block for postoperative analgesia after robotic-assisted coronary artery bypass surgery. Ann Card Anaesth 2008; 11 : 91-6.

6. Kot P, Rodriguez P, Granell M, et al. The erector spinae plane block: a narrative review. Korean J Anesthesiol 2019; 72: 209-20.

7. Nagaraja PS, Ragavendran S, Singh NG, et al. Comparison of continuous thoracic epidural analgesia with bilateral erector spinae plane block for perioperative pain management in cardiac surgery. Ann Card Anaesth 2018; 21: 323-7.
8. Bigeleisen PE, Goehner N. Novel approaches in pain management in cardiac surgery. Curr Opin Anaesthesiol 2015; 28: 89-94.

9. Naveed A, Azam H, Murtaza HG, et al. Incidence and risk factors of pulmonary complications after cardiopulmonary bypass. Pakistan J Med Sci 2017; 33: 993-6.

10. Khera T, Murugappan KR, Leibowitz A, et al. UltrasoundGuided Pecto-Intercostal Fascial Block for Postoperative Pain Management in Cardiac Surgery: A Prospective, Randomized, Placebo-Controlled Trial. J Cardiothorac Vasc Anesth 2021; 35 . 896-903

11.Doğan Bakı E, Kavrut Ozturk N, Ayoğlu RU, Emmiler M, Karslı B, Uzel H. Effects of parasternal block on acute and chronic pain in patients undergoing coronary artery surgery. Seminars in Cardiothoracic and Vascular Anesthesia 2016; 20: 205-12.

12. Macaire P, Ho N, Nguyen T, et al. Ultrasound-guided continuous thoracic erector spinae plane block within an enhanced recovery program is associated with decreased opioid consumption and improved patient postoperative rehabilitation after open cardiac surgery-a patient-matched, controlled before-and-after study. J Cardiothorac Vasc Anesth 2019; 33: 1659-67.

13. Krishna SN, Chauhan S, Bhoi D, et al. Bilateral erector spinae plane block for acute post-surgical pain in adult cardiac surgical patients: a randomized controlled trial. J Cardiothorac Vasc Anesth 2019; 33: 368-75.

14. Athar M, Parveen S, Yadav M, et al. A randomized double-blind controlled trial to assess the efficacy of ultrasound-guided erector spinae plane block in cardiac surgery. J Cardiothorac Vasc Anesth 2021; S1053-0770(21)00211-1

15. Svircevic V, van Dijk D, Nierich AP, et al. Meta-analysis of thoracic epidural anesthesia versus general anesthesia for cardiac surgery. Anesthesiology 2011; 114: 271-82.

16. Wilson JM, Lohser J, Klaibert B. Erector spinae plane block for postoperative rescue analgesia in thoracoscopic surgery. J Cardiothorac Vasc Anesth 2018; 32: e5-7.

17. Luis-Navarro JC, Seda-Guzmán M, Luis-Moreno C, LópezRomero JL. The erector spinae plane block in 4 cases of videoassisted thoracic surgery. Rev Esp Anestesiol Reanim 2018; 65: 204-8.

18. Adhikary SD, Prasad A, Soleimani B, Chin KJ. Continuous erector spinae plane block as an effective analgesic option in anticoagulated patients after left ventricular assist device implantation: a case series. J Cardiothorac Vasc Anesth 2019; 33: 1063-7.

19. Wyatt K, Elattary T. The erector spinae plane block in a highrisk Ehlers-Danlos syndrome pediatric patient for vascular ring repair. J Clin Anesth 2019; 54: 39-40.

20. Ueshima H. Pneumothorax after the erector spinae plane block. J Clin Anesth 2018; 48: 12.

21. Hamilton DL. Pneumothorax following erector spinae plane block. J Clin Anesth 2019; 52: 17.

22. Huang J, Liu JC. Ultrasound-guided erector spinae plane block for postoperative analgesia: a meta-analysis of randomized controlled trials. BMC Anesthesiol 2020; 20: 83.

23. Taketa Y, Irisawa Y, Fujitani T. Ultrasound-guided erector spinae plane block elicits sensory loss around the lateral, but not the parasternal, portion of the thorax. J Clin Anesth 2018; 47: 84-5.

24. Ueshima H, Hiroshi O. Spread of local anesthetic solution in the erector spinae plane block. J Clin Anesth 2017; 45: 23 